\begin{tabular}{|c|l|}
\hline Title & Effects of surface roughness on the paramagnetic response of small unconventional superconductors \\
\hline Author(s) & Suzuki, Shu-Ichiro; A sano, Y asuhiro \\
\hline Citation & $\begin{array}{l}\text { Physical review B, 91(21), 214510 } \\
\text { https://doi.org/L0.1103/PhysRevB.91.214510 }\end{array}$ \\
\hline Issue Date & 2015-06-18 \\
\hline Doc URL & http://hdl.handle.net/2115/59554 \\
\hline Rights & @2015A American Physical Society \\
\hline Type & article \\
\hline File Information & PhysRevB.91.214510.pdf \\
\hline
\end{tabular}

Instructions for use 


\title{
Effects of surface roughness on the paramagnetic response of small unconventional superconductors
}

\author{
Shu-Ichiro Suzuki ${ }^{1}$ and Yasuhiro Asano ${ }^{1,2,3}$ \\ ${ }^{1}$ Department of Applied Physics, Hokkaido University, Sapporo 060-8628, Japan \\ ${ }^{2}$ Center for Topological Science \& Technology, Hokkaido University, Sapporo 060-8628, Japan \\ ${ }^{3}$ Moscow Institute of Physics and Technology, 141700 Dolgoprudny, Russia \\ (Received 15 April 2015; revised manuscript received 21 May 2015; published 17 June 2015)
}

\begin{abstract}
We theoretically study the effects of surface roughness on the magnetic response of small unconventional superconductors by solving the Eilenberger equation for the quassiclassical Green function and the Maxwell equation for the vector potential simultaneously and self-consistently. The paramagnetic phase of spin-singlet $d$-wave superconducting disks is greatly suppressed by the surface roughness, whereas that of spin-triplet $p$-wave disks is robust even in the presence of the roughness. This difference derives from the orbital symmetry of paramagnetic odd-frequency Cooper pairs appearing at the surface of the disks. The orbital part of the paramagnetic pairing correlation has $p$-wave symmetry in the $d$-wave disks, whereas it has $s$-wave symmetry in the $p$-wave disks. Calculating the free energy, we also confirm that the paramagnetic state is more stable than the normal state, which indicates a possibility of detecting the paramagnetic effect in experiments. Indeed, our results are consistent with an experimental finding on high- $T_{c}$ thin films.
\end{abstract}

DOI: 10.1103/PhysRevB.91.214510

PACS number(s): 73.20.At, 73.20.Hb

\section{INTRODUCTION}

A diamagnetic response to an external magnetic field is a fundamental property of all superconductors [1]. The Meissner current (coherent motion of the Cooper pairs) screens a weak magnetic field at the surface of a superconductor. As a result, the phase coherence of the superconducting condensate is well preserved far away from the surface. A number of experiments, however, have reported the paramagnetic response of small superconductors and mesoscopic proximity structures [2-7].

Recent theoretical studies have suggested the existence of paramagnetic Cooper pairs in inhomogeneous superconductors [8-13]. A spatial gradient of the superconducting order parameter induces subdominant pairing correlations. The pairing symmetry of such induced Cooper pairs is different from that of the principal Cooper pairs in the bulk superconducting state [13-15]. For example, the principal Cooper pairs in high- $T_{c}$ superconductors belong to the spinsinglet $d$-wave (even-parity) class. In the (110) direction of high- $T_{c}$ cuprates, a surface acts as a pair breaker and greatly suppresses the pair potential. Simultaneously, spinsinglet odd-parity pairs are locally induced at the surface as a subdominant correlation. A surface generates odd-parity pairing correlations from the $d$-wave even-parity correlation because the surface breaks inversion symmetry locally. Since the pairing correlation function must be antisymmetric under the permutation of two electrons, the induced pairs have oddfrequency symmetry [16]. To our knowledge, such induced odd-frequency pairs indicate a paramagnetic response to an external magnetic field. Odd-frequency Cooper pairs can be generated also from conventional superconductors in the presence of spin-dependent potentials [17].

In a previous paper [18], we showed that the magnetic susceptibility of small enough unconventional superconducting disks can be paramagnetic at a sufficiently low temperature. Odd-frequency Cooper pairs induced by a surface are responsible for the unusual paramagnetic Meissner effect. The magnetic response of Cooper pairs is well characterized by the so-called "pair density," which is defined by diagonal elements of the response function to a magnetic field. Even-frequency Cooper pairs have a positive pair density, whereas induced odd-frequency pairs have a negative pair density. So far an experiment has reported the decrease of the pair density at low temperature in high- $T_{c}$ superconducting films on which internal surfaces are introduced by heavy-ion irradiation [19]. Thus our theoretical results are consistent with the experiment at least qualitatively. However, the signs of the paramagnetic effect in the experiment [19] are much weaker than our theoretical prediction. The discrepancy may come from the sample quality at surfaces. Artificially introduced internal surfaces can be very rough in experiments, whereas the surfaces are specular in the theory. Actually, several theories have pointed out that the surface roughness affects properties of the surface Andreev bound states of a high- $T_{c}$ superconductor [20-24].

The purpose of this paper is to clarify the effects of surface roughness on the paramagnetic Meissner response of small unconventional superconductors. We consider a twodimensional superconducting disk with spin-singlet $d$-wave or spin-triplet $p$-wave symmetry. In a numerical simulation, we solve the Eilenberger equation and the Maxwell equation simultaneously and self-consistently. Surface roughness is considered through an impurity self-energy within the selfconsistent Born approximation. We find that the surface roughness greatly suppresses the paramagnetic response of a spin-singlet $d$-wave superconducting disk. On the other hand in a spin-triplet $p$-wave disk, the paramagnetic property is robust even in the presence of surface roughness. The induced odd-frequency pairing correlation has $p$-wave symmetry in the former, whereas it has $s$-wave symmetry in the latter. In addition, we also confirm that the paramagnetic superconducting states are more stable than the normal state by calculating the free energies.

This paper is organized as follows. In Sec. II, we explain the theoretical method used to analyze the magnetic response of small superconducting disks. In Sec. III, we discuss the magnetic response of small superconducting disks with a rough surface. In Sec. IV, we consider the stability of a paramagnetic 
state by calculating the free energies of the superconducting state. We summarize this paper in Sec. V.

\section{FORMULATION}

Let us consider a superconducting disk in two dimensions as shown in Fig. 1(a), where $R$ is the radius of the disk. To describe rough surfaces, we introduce random impurity potentials near the surface. The width of the disordered region $w$ is measured from the surface as shown in Fig. 1(a). An external magnetic field $H^{\text {ext }}$ is applied in the $z$ direction. Throughout this paper, we use units of $\hbar=c=k_{B}=1$ with $k_{B}$ and $c$ being the Boltzmann constant and the speed of light, respectively.

Superconducting states in equilibrium are described by solutions of the quasiclassical Eilenberger equation [25],

$$
i v_{F} \boldsymbol{k} \cdot \nabla_{\boldsymbol{r}} \check{g}+[\check{H}+\check{\Sigma}, \check{g}]=0,
$$

where $v_{F}$ is the Fermi velocity, and $\boldsymbol{k}$ is a unit vector on the Fermi surface. $\breve{g}$ and $\breve{H}$ are defined as follows:

$$
\begin{gathered}
\check{g}\left(\boldsymbol{r}, \boldsymbol{k}, i \omega_{n}\right)=\left[\begin{array}{cc}
\hat{g}\left(\boldsymbol{r}, \boldsymbol{k}, i \omega_{n}\right) & \hat{f}\left(\boldsymbol{r}, \boldsymbol{k}, i \omega_{n}\right) \\
-\hat{f}\left(\boldsymbol{r}, \boldsymbol{k}, i \omega_{n}\right) & -\underset{\sim}{\hat{g}}\left(\boldsymbol{r}, \boldsymbol{k}, i \omega_{n}\right)
\end{array}\right], \\
\check{H}\left(\boldsymbol{r}, \boldsymbol{k}, i \omega_{n}\right)=\left[\begin{array}{cc}
\hat{\xi}\left(\boldsymbol{r}, \boldsymbol{k}, i \omega_{n}\right) & \hat{\Delta}(\boldsymbol{r}, \boldsymbol{k}) \\
\hat{\sim}(\boldsymbol{r}, \boldsymbol{k}) & \underset{\sim}{\hat{\xi}}\left(\boldsymbol{r}, \boldsymbol{k}, i \omega_{n}\right)
\end{array}\right], \\
\hat{\xi}\left(\boldsymbol{r}, \boldsymbol{k}, i \omega_{n}\right)=\left[i \omega_{n}+e v_{F} \boldsymbol{k} \cdot \boldsymbol{A}(\boldsymbol{r})\right] \hat{\sigma}_{0},
\end{gathered}
$$

where $\omega_{n}=(2 n+1) \pi T$ is the fermionic Matsubara frequency, $n$ is an integer number, and $T$ is the temperature. In this paper, the symbol $`$ represents a $4 \times 4$ matrix structure, $\hat{\imath}$ represents a $2 \times 2$ matrix structure in spin space, and $\hat{\sigma}_{0}$ is the identity matrix in spin space. The vector potential is denoted by $\boldsymbol{A}(\boldsymbol{r})$. We introduced the definition $\underset{\sim}{K}\left(\boldsymbol{r}, \boldsymbol{k}, i \omega_{n}\right) \equiv$

$K^{*}\left(\boldsymbol{r},-\boldsymbol{k}, i \omega_{n}\right)$ for all functions $K\left(\boldsymbol{r}, \boldsymbol{k}, i \omega_{n}\right)$. The effects of rough surfaces are taken into account through the impurity self-energy of a quasiparticle defined by

$$
\check{\Sigma}\left(\boldsymbol{r}, i \omega_{n}\right)=\Theta(|\boldsymbol{r}|-R+w) \frac{i}{2 \tau_{0}} \int \frac{d \boldsymbol{k}}{2 \pi} \check{g}\left(\boldsymbol{r}, \boldsymbol{k}, i \omega_{n}\right),
$$

where $\tau_{0}$ is the lifetime of the quasiparticle and $\Theta(x)$ is the Heaviside step function. The mean free path of the quasiparticle is defined by $\ell=v_{F} \tau_{0}$ in the disordered region.
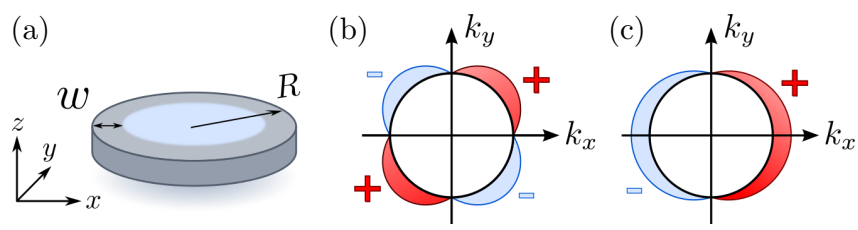

FIG. 1. (Color online) (a) Schematic figure of a superconducting disk with a rough surface, with $R$ and $w$ the radius of the disk and the width of the disordered region, respectively. An external magnetic field is applied in the $z$ direction. The origin of a spatial coordinate is located at the center of the disk. The pair potential in momentum space for a $d$-wave superconductor and that for a $p$-wave one are illustrated in (b) and (c), respectively.
The anomalous Green function $\hat{f}\left(\boldsymbol{r}, \boldsymbol{k}, i \omega_{n}\right)$ is defined by the average of two annihilation operators of an electron. The relation

$$
\hat{f}\left(\boldsymbol{r}, \boldsymbol{k}, i \omega_{n}\right)=-\hat{f}^{\mathrm{T}}\left(\boldsymbol{r},-\boldsymbol{k},-i \omega_{n}\right)
$$

represents the antisymmetric property of the anomalous Green function under the permutation of two electrons, where $\mathrm{T}$ represents the transpose of a matrix.

The direction of $\boldsymbol{k}$ in two-dimensional momentum space is represented by the angle $\theta$ measured from the $x$ axis (i.e., $k_{x}=\cos \theta$ and $k_{y}=\sin \theta$ ). In what follows, we consider two unconventional superconductors with different pairing symmetries. One has spin-singlet $d$-wave symmetry $\hat{\Delta}(\boldsymbol{r}, \theta)=$ $\Delta(\boldsymbol{r}) \sin (2 \theta) i \hat{\sigma}_{2}$. The other has spin-triplet $p$-wave symmetry $\hat{\Delta}(\boldsymbol{r}, \theta)=\Delta(\boldsymbol{r}) \cos (\theta) \hat{\sigma}_{1}$, where $\hat{\sigma}_{j}$ for $j=1-3$ are the Pauli matrices in spin space. A $d$-wave and a $p$-wave pair potential in momentum space are shown schematically in Figs. 1(b) and 1(c), respectively. We do not consider any spin-dependent potentials in this paper. The matrix structure of the Green function is represented by

$$
\begin{aligned}
& \hat{g}\left(\boldsymbol{r}, \theta, i \omega_{n}\right)=g\left(\boldsymbol{r}, \theta, i \omega_{n}\right) \hat{\sigma}_{0}, \\
& \hat{f}\left(\boldsymbol{r}, \theta, i \omega_{n}\right)=f\left(\boldsymbol{r}, \theta, i \omega_{n}\right) \times \begin{cases}\hat{\sigma}_{2} & \text { for a spin singlet, } \\
-i \hat{\sigma}_{1} & \text { for a spin triplet, }\end{cases}
\end{aligned}
$$

with scalar Green functions $g\left(\boldsymbol{r}, \theta, i \omega_{n}\right)$ and $f\left(\boldsymbol{r}, \theta, i \omega_{n}\right)$. The spatial dependence of $\Delta(\boldsymbol{r})$ is determined self-consistently from the gap equation

$$
\Delta(\boldsymbol{r})=\pi N_{0} g_{0} T \sum_{\omega_{n}} \int \frac{d \theta}{2 \pi} f\left(\boldsymbol{r}, \theta, i \omega_{n}\right) V_{x}(\theta),
$$

where $N_{0}$ is the density of states per spin of a normal metal at the Fermi level, $g_{0}$ is the coupling constant, and $V_{x}$ represents attractive electron-electron interactions with $x=p$ wave or $d$ wave indicating the pairing symmetries. The interaction kernel $V_{x}$ depends upon the pairing symmetries as

$$
V_{x}(\theta)= \begin{cases}2 \cos \theta & \text { for } \quad x=p \text { wave, } \\ 2 \sin (2 \theta) & \text { for } \quad x=d \text { wave. }\end{cases}
$$

The constant $N_{0} g_{0}$ is determined by

$$
\left(N_{0} g_{0}\right)^{-1}=\ln \left(\frac{T}{T_{c}}\right)+\sum_{0 \leqslant n<\omega_{c} / 2 \pi T} \frac{1}{n+1 / 2},
$$

with $T_{c}$ and $\omega_{c}$ being the transition temperature and the cutoff energy, respectively.

In a type-II superconductor, an electric current is represented by

$$
\boldsymbol{j}(\boldsymbol{r})=\frac{\pi e v_{F} N_{0}}{2 i} T \sum_{\omega_{n}} \int \frac{d \theta}{2 \pi} \operatorname{Tr}\left[\check{T}_{3} \boldsymbol{k} \check{g}\left(\boldsymbol{r}, \theta, \omega_{n}\right)\right],
$$

with $\check{T}_{3}=\operatorname{diag}\left[\hat{\sigma}_{0},-\hat{\sigma}_{0}\right]$. From Eq. (12) and the Maxwell equation $\nabla \times \boldsymbol{H}(\boldsymbol{r})=4 \pi \boldsymbol{j}(\boldsymbol{r})$, we obtain the spatial profiles of the vector potential $\boldsymbol{A}(\boldsymbol{r})$ and the local magnetic field $\boldsymbol{H}(\boldsymbol{r})$. The local magnetic susceptibility is defined by

$$
\chi(\boldsymbol{r})=\frac{1}{4 \pi} \frac{H(\boldsymbol{r})-H^{\mathrm{ext}}}{H^{\mathrm{ext}}},
$$


where $H^{\text {ext }}$ is the amplitude of an external magnetic field applied in the $z$ direction. By integrating the local susceptibility, we obtain the susceptibility of the whole disk $X$ as

$$
X=\frac{1}{\pi R^{2}} \int_{|\boldsymbol{r}| \leqslant R} d \boldsymbol{r} \chi(\boldsymbol{r}) .
$$

To solve the Eilenberger equation (1) in a disk geometry, we use a Riccati parametrization [26-28] and a numerical method discussed in Ref. [29]. Using this parametrization, the Eilenberger equation can be separated into two Riccati-type differential equations. When we solve the Riccati-type equation along a long enough quasiclassical trajectory, solutions of the equation do not depend on the initial conditions [29]. In this paper, the length of the classical trajectories is more than 30 times the coherence length. Solving the Eilenberger equation and the Maxwell equation, we determine the pair potential, the vector potential, and the self-energy self-consistently with one another. At surfaces, we consider specular classical trajectories for calculating the Green functions [29]. The vector potential outside the superconducting disk is $\boldsymbol{A}(\boldsymbol{r})=\left(H^{\mathrm{ext}} / 2\right)(-y \hat{\boldsymbol{x}}+$ $x \hat{\boldsymbol{y}}$ ) which gives a uniform magnetic field in the $z$ direction, where $\boldsymbol{x}$ and $\boldsymbol{y}$ are the unit vectors in the $x$ and $y$ directions, respectively.

\section{RESULTS}

Throughout this paper, we fix several parameters as $R=$ $10 \xi_{0}$ and $\omega_{c}=10 \Delta_{0}$, where $\Delta_{0}$ is the amplitude of the pair potential at zero temperature, and $\xi_{0}=\hbar v_{F} / 2 \pi T_{c}$ is the coherence length. The strength of the disorder is tuned by changing the parameter $\xi_{0} / \ell$. The width of the disordered region is $w=3 \xi_{0}$ because odd-frequency pairs induced by a surface are localized within this range. All lengths are

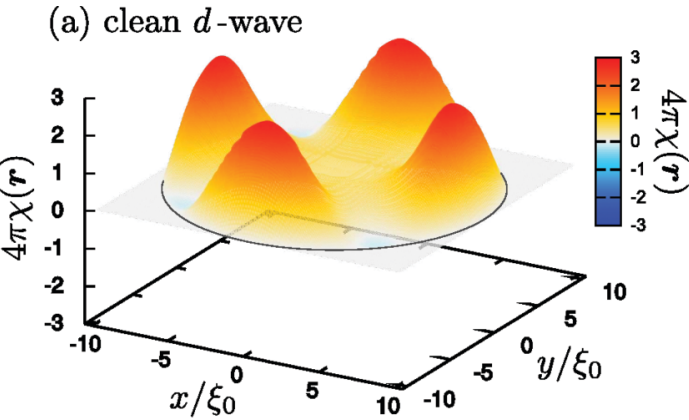

(c) disordered $d$-wave

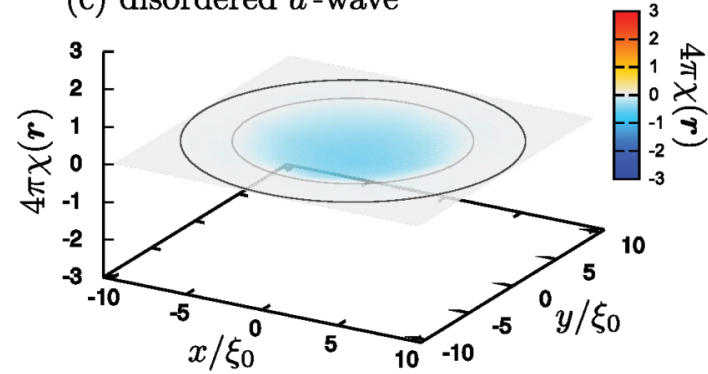

measured in units of $\xi_{0}$. The current density is normalized to $J_{0}=\hbar c^{2} / 4 \pi|e| \xi_{0}^{3}$. Here we express $\hbar$ and $c$ explicitly to avoid misunderstandings. The characteristic length scale of the Maxwell equation is the penetration depth defined as $\lambda_{L}=\left(4 \pi n e^{2} / m c^{2}\right)^{-1 / 2}$ and is a parameter in numerical simulations. In this paper, we choose $\lambda_{L}=5 \xi_{0}$ to realize type-II superconductors and fix $H^{\text {ext }}=0.01 H_{c_{2}}$. Here $H_{c_{2}}=$ $\hbar c /|e| \xi_{0}^{2}$ is the second critical magnetic field. The first critical magnetic field at low temperature is estimated as $H_{c 1}=$ $H_{c_{2}}\left(\xi_{0} / \lambda_{L}\right)^{2} \ln \left(\lambda_{L} / \xi_{0}\right) \approx 0.03 H_{c 2}>H^{\text {ext }}$ at $\lambda_{L}=5 \xi_{0}$. Thus vortices are not expected at low temperatures. We start the numerical simulations with initial conditions of a spatially uniform pair potential $\Delta(\boldsymbol{r})=\left|\Delta_{\text {bulk }}(T)\right|$ and a homogeneous magnetic field $\boldsymbol{A}(\boldsymbol{r})=\left(H^{\text {ext }} / 2\right)(-y \hat{\boldsymbol{x}}+x \hat{\boldsymbol{y}})$, where $\Delta_{\text {bulk }}(T)$ is the pair potential obtained in a homogeneous superconductor at a temperature $T$. If we choose an alternative initial condition hosting a vortex in a superconductor, a vortex state might be realized in numerical simulations [30] even for $H^{\text {ext }}<H_{c 1}$. This vortex issue, however, goes beyond the scope of this paper.

First we discuss the calculated results for a small superconducting disk with a specular surface (i.e., $w=0, \xi_{0} / \ell=0$ ). Then we discuss the effects of surface roughness by comparing the numerical results in a disk with a rough surface with those with a specular disk.

\section{A. Disks with a specular surface}

We briefly explain the paramagnetic Meissner effect in a superconducting disk with a specular surface. Figure 2(a) shows the local susceptibility in a $d$-wave superconductor. The result are fourfold symmetric, reflecting the $d$-wave pair potential. The magnetic susceptibility is positive (paramagnetic) near

(b) clean $p$-wave

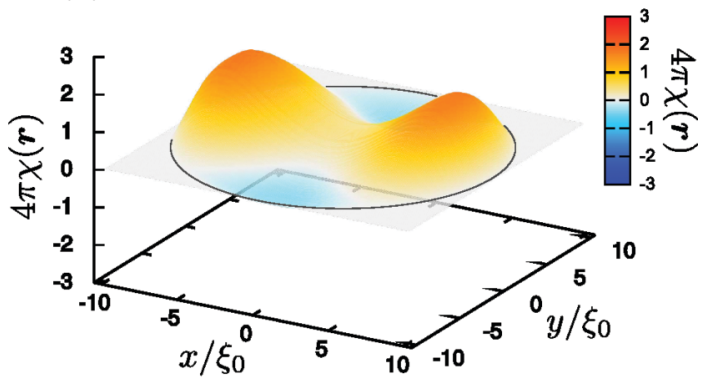

(d) disordered $p$-wave

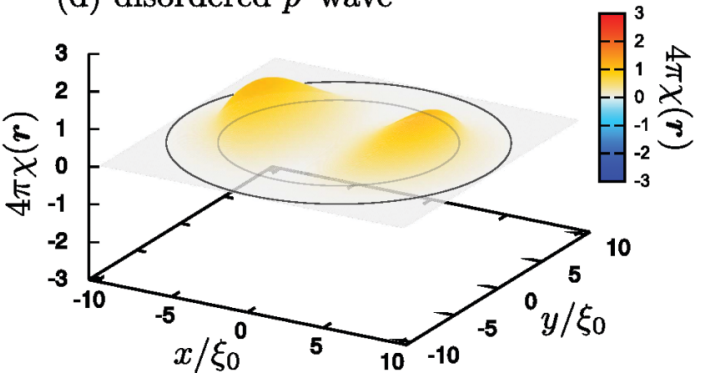

FIG. 2. (Color) Local susceptibilities of small superconducting disks. The results for a $d$-wave and those for a $p$-wave superconductor with a clean surface (i.e., $w=0, \xi / \ell=0$ ) are presented in (a) and (b), respectively. The results for a $d$-wave and those for a $p$-wave superconductor with a rough surface $\left(w=3 \xi_{0}, \xi_{0} / \ell=1.0\right)$ are demonstrated in (c) and (d), respectively. The parameters used in the simulation are $R=10 \xi_{0}$, $\lambda_{L}=5 \xi_{0}, \omega_{c}=10 \Delta_{0}, H^{\mathrm{ext}}=0.01 H_{c_{1}}$, and $T=0.1 T_{c}$. 

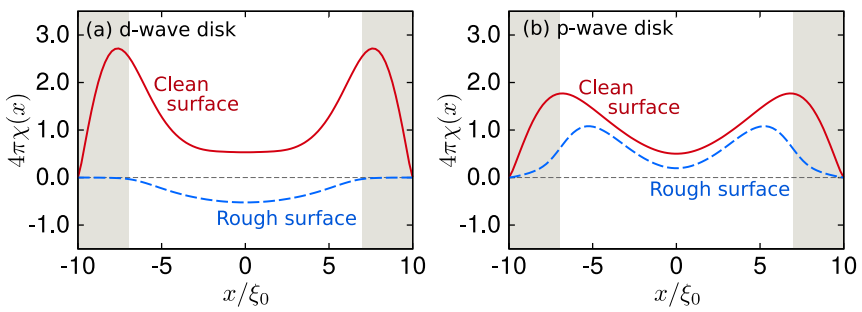

FIG. 3. (Color online) Local susceptibilities on the $x$ axis (i.e., $y=0$ ). The results for a $d$-wave disk and for a $p$-wave disk are plotted in (a) and (b), respectively. All the parameters are set to be the same as those of Fig. 2. The solid and the broken lines indicate the results for the disk with the clean surface and those with the rough surface, respectively. The shadowed areas indicate the disordered regions.

four surfaces in the $x$ and the $y$ directions. In Fig. 3(a), we show a spatial profile of the local susceptibility of Fig. 2(a) along the $x$ axis at $y=0$. We also show the amplitude of the pair potential in real space $|\Delta(\boldsymbol{r})|$ in Fig. 4(a). The pair potential is calculated along a trajectory $\rho_{\alpha}$ oriented at an angle $\alpha$ measured from the $x$ axis as shown in Fig. 4. In a $d$-wave disk, the pair potential is fourfold symmetric. The pair potential is strongly suppressed at the four surfaces in the $x$ and $y$ directions as a result of the surface Andreev bound states that appear [31], whereas it is totally constant in the directions in which the surface Andreev bound states are absent. In contrast to the pair potential around a vortex core [30], the results in Fig. 4(a) are anisotropic in real space. As shown in Ref. [13],

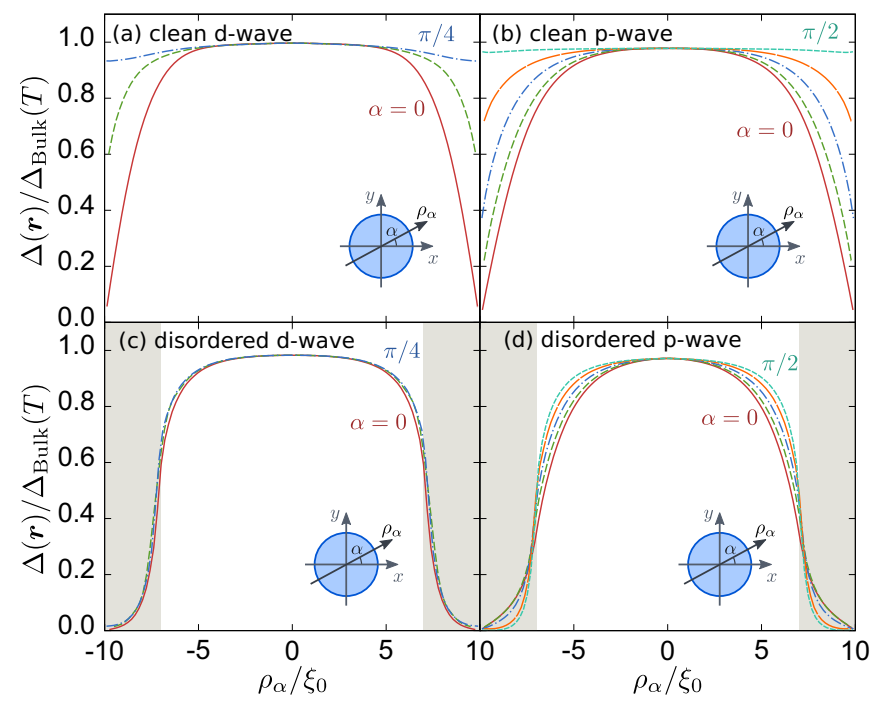

FIG. 4. (Color online) Amplitudes of pair potentials in real space for several azimuthal angles $\alpha$ measured from the $x$ axis. The results for a $d$-wave disk with $\ell / \xi_{0}=0.0$ and $\ell / \xi_{0}=1.0$ are shown in (a) and (c), respectively. Since the pair potential is fourfold symmetric, we plot its spatial profile for $\alpha=0, \pi / 8$, and $\pi / 4$. The results for a $p$-wave disk with $\ell / \xi_{0}=0.0$ and $\ell / \xi_{0}=1.0$ are shown in (b) and (d), respectively. The pair potentials for $\alpha=0, \pi / 8, \pi / 4,3 \pi / 8, \pi / 2$ (from bottom to top) are shown since the results are twofold symmetric. The pair potentials are normalized to $\Delta_{\text {bulk }}(T)$. All the parameters are set to be the same as those of Fig. 2. The shadowed areas in (c) and (d) indicate the disordered regions.

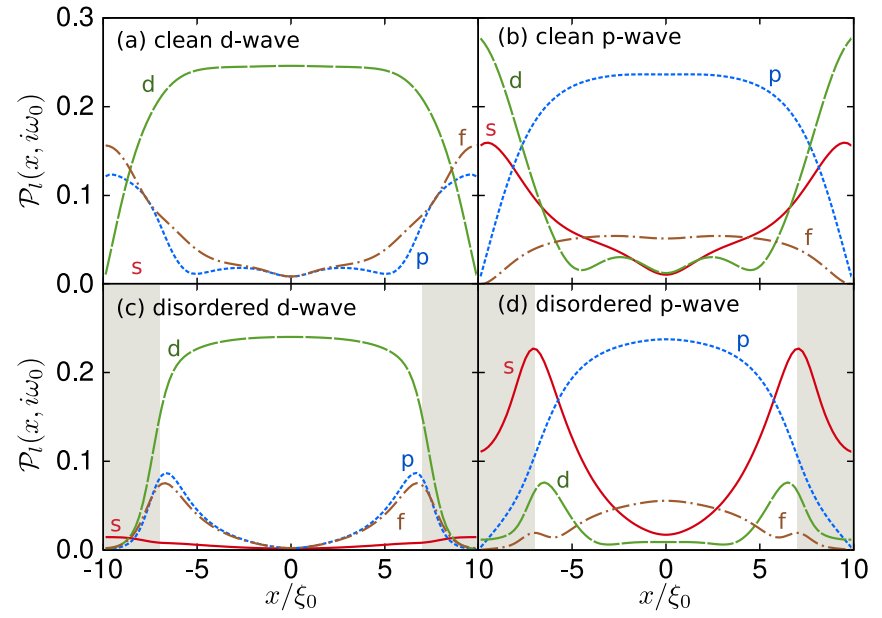

FIG. 5. (Color online) Spatial dependencies of the decomposed pairing functions at the lowest Matsubara frequency on the $x$ axis for (a) a clean $d$-wave, (b) a clean $p$-wave, (c) a disordered $d$-wave, and (d) a disordered $p$-wave disk. The parameters are set to the same values as those in Fig. 2. The shadowed areas indicate the disordered regions.

the spatial gradient of the pair potential generates paramagnetic pairing correlations with odd-frequency symmetry. Thus oddfrequency pairs are expected at four surfaces in the $x$ and $y$ directions, which explains the inhomogeneous and angular anisotropic paramagnetic response in Fig. 2(a). In this paper, we analyze the frequency symmetries of Cooper pairs by decomposing the pairing functions into a series of Fourier components. In a $d$-wave disk, the anomalous Green functions are described by two components

$$
\hat{f}\left(\boldsymbol{r}, \theta, i \omega_{n}\right)=\left[f_{\mathrm{ep}}\left(\boldsymbol{r}, \theta, i \omega_{n}\right)+f_{\mathrm{op}}\left(\boldsymbol{r}, \theta, i \omega_{n}\right)\right] \hat{\sigma}_{2},
$$

where $f_{\text {ep }}$ is an even-parity ( $d$-wave) function representing the principal pairing correlation and $f_{\text {op }}$ is an odd-parity function representing the induced pairing component at the surface. To satisfy Eq. (6), $f_{\text {op }}$ must be an odd function of $\omega_{n}$. We decompose the pair functions $f\left(\boldsymbol{r}, \theta, i \omega_{n}\right)$ as

$$
\begin{gathered}
\mathcal{P}_{l}\left(\boldsymbol{r}, i \omega_{n}\right)=2 \sqrt{C_{l}^{2}+S_{l}^{2}}, \\
S_{l}=\int \frac{d \theta}{2 \pi} \operatorname{Re}\left[f\left(\boldsymbol{r}, \theta, i \omega_{n}\right)\right] \sin (l \theta), \\
C_{l}=\int \frac{d \theta}{2 \pi} \operatorname{Re}\left[f\left(\boldsymbol{r}, \theta, i \omega_{n}\right)\right] \cos (l \theta),
\end{gathered}
$$

where $l=0,1,2$, and 3 correspond to $s^{-}, p-, d$-, and $f$-wave orbital functions, respectively. In the presence of a magnetic field, the imaginary part of $f\left(\boldsymbol{r}, \theta, i \omega_{n}\right)$ is induced by the vector potential as analytically shown in the Appendix. We focus only on the real part of $f$ to analyze the pairing symmetries. Figure 5(a) indicates the spatial profile of $\mathcal{P}_{l}\left(x, i \omega_{0}\right)$ at the lowest Matsubara frequency as a function of $x$ at $y=0$. The pairing functions of induced Cooper pairs have $p$ and $f$-wave symmetries and their amplitudes are localized near the surface. Such odd-frequency Cooper pairs show a paramagnetic response to a magnetic field. The surface also generates spin-singlet $s$-wave correlations. Its amplitude, 
(a) clean $d$-wave

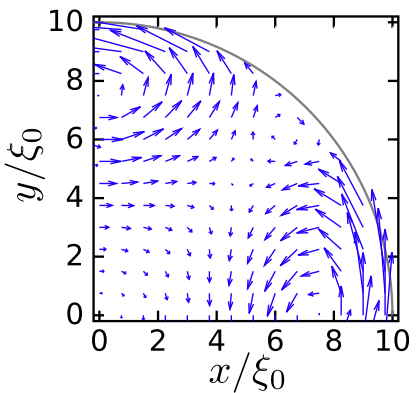

(c) disordered $d$-wave

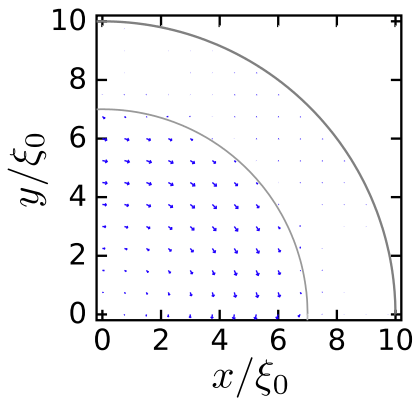

(b) clean $p$-wave

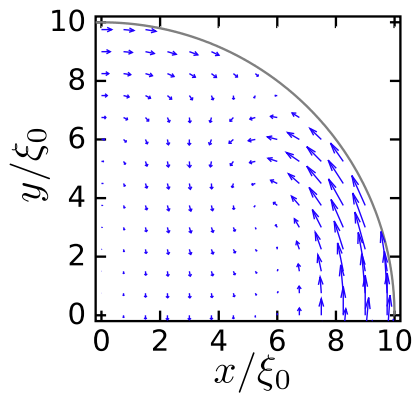

(d) disordered $p$-wave

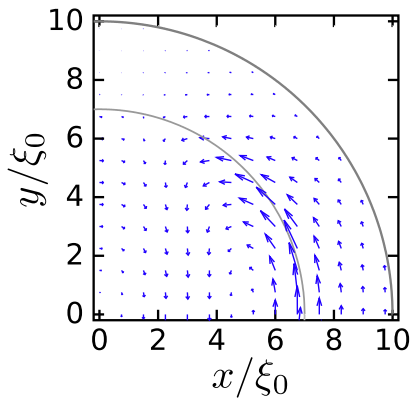

FIG. 6. (Color online) Spatial distribution of the current density on a superconducting disk. The results for a $d$-wave and a $p$-wave superconductor with a clean surface $(w=0, \xi / \ell=0.0)$ are shown in (a) and (b), respectively. The results with a rough surface are shown in (c) and (d). The outer circles indicate the edge of the disk and the inner circles in (c) and (d) indicate the effective interface between the clean region and the disordered region. The parameters are set to the same values as those in Fig. 2.

however, is too small to confirm this at the scale of the plot in Fig. 5(a). Figure 6(a) shows the spatial distribution of the electric current on a $d$-wave disk. A diamagnetic current flows in the central region because of the usual Meissner effect. Near the surfaces in the $x$ and $y$ directions, however, the current flows in the opposite direction to the Meissner current. Therefore a small $d$-wave superconductor can be paramagnetic due to the induced odd-frequency Cooper pairs at its surface.

A spin-triplet $p$-wave disk also indicates a similar paramagnetic effect as shown by the results for the magnetic susceptibility in Fig. 2(b), its spatial profile on the $x$ axis in Fig. 3(b), the pair potential in Fig. 4(b), and the electric current in Fig. 6(b). The results are twofold symmetric, reflecting the $p$-wave superconducting pair potential. The paramagnetic effect can be seen near the surface in the $x$ direction because of the induced odd-frequency Cooper pairs. The anomalous Green function is represented by Eq. (15) with the replacement of $\hat{\sigma}_{2}$ by $-i \hat{\sigma}_{1}$. In the $p$-wave case, $f_{\text {ep }}$ represents induced pairing correlations and is an odd function of $\omega_{n}$. As shown in Fig. 5(b), $f_{\text {ep }}$ mainly consists of $s$ - and $d$-wave pairing correlations.

Finally in this section, we summarize an important difference between the paramagnetic effects of a $d$-wave and of a $p$-wave superconductor. In a $d$-wave disk, the surface odd-frequency Cooper pairs have $p$ - or $f$-wave symmetry $[15,18]$. In a $p$-wave disk, on the other hand, $s$ - or

$d$-wave odd-frequency Cooper pairs are responsible for the paramagnetic effect $[15,18]$. In the next section, we will show that the paramagnetic response of a disk with a rough surface depends sensitively on the orbital symmetry of the induced odd-frequency pairs at the surface.

\section{B. Disks with a rough surface}

Next, we discuss the effects of the surface roughness on the magnetic response of a small superconductor. The calculated results for the local susceptibility for a $d$-wave superconducting disk with a rough surface are shown in Fig. 2(c), where we choose $\xi_{0} / \ell=1.0$. Comparing Fig. 2(a) with Fig. 2(c), we find that the surface roughness completely suppresses the paramagnetic response at the four surfaces in a $d$-wave disk. The central region of the disk with the rough surface recovers the usual diamagnetic response. This effect is demonstrated more clearly in the spatial profile of local susceptibilities at $y=0$ in Fig. 3(a), where the shadowed area indicates the disordered region. The amplitude of the pair potential in real space is shown in Fig. 4(c). The pair potential in the disordered region is totally suppressed because the random impurity potential acts as a pair breaker for $d$-wave Cooper pairs. Spatial profiles of the decomposed pairing functions are shown in Fig. 5(c). In the disordered region, a $d$-wave pairing function $\mathcal{P}_{d}$ is drastically suppressed due to impurity scattering. The disordered region can be considered as a diffusive normal metal because the spatial profile of the order parameter is proportional to $\mathcal{P}_{d}$. Oddfrequency Cooper pairs are also fragile in the presence of surface roughness because they have $p$ - or $f$-wave pairing symmetry. Therefore, both the paramagnetic and the diamagnetic currents disappear in the disordered region as shown in Fig. 6(c). The magnetic property of a disk is determined by that in the clean central region where even-frequency $d$-wave Cooper pairs remain and contribute to the diamagnetic response. We conclude that the paramagnetic effect in a $d$-wave disk is fragile in the presence of surface roughness because odd-frequency pairs have $p$-wave or $f$-wave orbital symmetry.

A $p$-wave disk shows a qualitatively different magnetic response from a $d$-wave one. The local susceptibility of a $p$ wave disk with a rough surface is shown in Figs. 2(d) and 3(b) with a broken line. Although the surface is rough enough, a $p$ wave superconducting disk still shows a strong paramagnetic response. The peak of $\chi$ in Fig. 3(b) shifts to the inside of the disk in the presence of surface roughness. This suggests that Andreev bound states appear at the boundary between the clean central region and the disordered surface region. Such Andreev bound states always accompany paramagnetic odd-frequency Cooper pairs. In addition to this, the paramagnetic response in Fig. 3(b) suggests the penetration of odd-frequency Cooper pairs into the surface disordered region. The spatial profile of the electric current in Fig. 6(d) shows that the paramagnetic current flows not only in the clean region but also in the disordered one. Odd-frequency pairs in a $p$-wave disk survive even in the presence of surface roughness because they have $s$-wave orbital symmetry. Therefore, the paramagnetic effect in a $p$-wave disk is robust against surface roughness. 


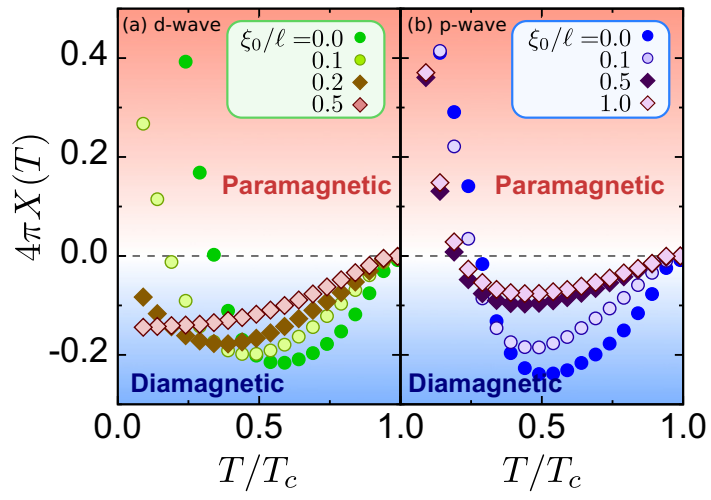

FIG. 7. (Color online) Temperature dependencies of the whole disk susceptibility $X(T)$ for the $d$-wave (a) and the $p$-wave (b) disk.

\section{Temperature dependence}

Here we discuss the magnetic susceptibility of the whole disk, which is a measurable value in experiments. The disk susceptibility in a $d$-wave and that in a $p$-wave superconductor is plotted as a function of temperature in Figs. 7(a) and 7(b), respectively. We present the results for several choices of the disorder $\xi_{0} / \ell$. In simulation, we first calculate the pair potential and the vector potential self-consistently at a temperature just below $T_{c}$ under an external magnetic field $H^{\text {ext }}=0.01 H_{c_{2}}$. Then the temperature is decreased while keeping $H^{\text {ext }}$ unchanged. In the clean limit $\left(\xi_{0} / \ell=0.0\right)$, both a $d$-wave and a $p$-wave disk show the usual diamagnetic response just below $T_{c}$. With decreasing temperature, however, the sign of the susceptibility changes around $T=T_{p} \sim 0.3 T_{c}$ for both cases. Here, we define $T_{p}$ as the diamagnetic-paramagnetic crossover temperature. Below $T_{p}$, superconducting disks show an anomalous paramagnetic response. The paramagnetic effect is stronger at lower temperatures because odd-frequency Cooper pairs are energetically localized at the Fermi level.

In a $d$-wave disk, the reentrance is slightly suppressed in the presence of moderate surface roughness with $\xi_{0} / \ell=0.1$ as shown in Fig. 7(a). When we increase the degree of roughness further, the paramagnetic response gradually becomes weaker. At $\xi_{0} / \ell=0.5$, the response is diamagnetic and the susceptibility recovers the monotonic temperature dependence which is usually observed in large enough superconductors in experiments. In $d$-wave superconductors, specular Andreev reflection is necessary for forming surface bound states at zero energy [32] and for the odd-frequency pairs that appear. In other words, odd-frequency pairs have $p$ - or $f$-wave orbital symmetry. Therefore the rough surface breaks oddfrequency pairs and suppresses the paramagnetic response. This conclusion is totally consistent with the experiment [19], where the temperature dependence of the pair density in a high- $T_{c}$ superconducting film shows a slight reentrant behavior at low temperature. But the total pair density remains positive. Actually, the experimental data are very similar to the results for $\xi_{0} / \ell=0.2$ in Fig. 7(a). The experimental results can be interpreted as the appearance of a small amount of oddfrequency pairs. In the experiment, the surface roughness may partially break odd-frequency pairs because a number of internal surfaces are introduced by the heavy-ion irradiation.
In contrast to $d$-wave disks, the susceptibility of a $p$ wave disk $X(T)$ shows reentrance and a crossover to the paramagnetic phase at low temperature for all $\xi_{0} / \ell$ as shown in Fig. 7(b). It has been pointed out that the surface Andreev bound states of a $p$-wave superconductor are robust under potential disorder because of the purely chiral property of surface bound states [33]. In other words, odd-frequency pairs accompanied by Andreev bound states have $s$-wave orbital symmetry $[15,18]$. Since $s$-wave pairs are robust under a disordered potential, the paramagnetic effect in $p$-wave superconductors persists even in the presence of surface roughness. We conclude that the robust paramagnetic response in a small size sample is a unique property of spin-triplet $p$-wave superconductors. This property would enable us to identify spin-triplet $p$-wave superconductivity in experiments.

\section{STABILITY OF PARAMAGNETIC SUPERCONDUCTING STATES}

Generally speaking, a superconducting phase is more stable than a normal one as long as the superconductor is diamagnetic and homogeneous [1]. Therefore a homogeneous paramagnetic superconducting phase is usually unstable. The calculated results in Sec. III, however, show that the paramagnetic phase on a small superconducting disk is spatially inhomogeneous. In this situation, it would be worth checking if the paramagnetic phase is a stable state at a free-energy minimum or a metastable state corresponding to a free-energy local minimum. In this section, we discuss the stability of the paramagnetic phase in small unconventional superconductors by calculating the free energy in clean superconducting disks.

The free energy is calculated from the quasiclassical Green functions [34]

$$
\begin{gathered}
F_{S}-F_{N}=\int d \boldsymbol{r} \mathcal{F}(\boldsymbol{r}), \\
\mathcal{F}(\boldsymbol{r})=\mathcal{F}_{\Delta}(\boldsymbol{r})+\mathcal{F}_{H}(\boldsymbol{r}), \\
\mathcal{F}_{H}(\boldsymbol{r})=\frac{\left\{H(\boldsymbol{r})-H^{\mathrm{ext}}\right\}^{2}}{8 \pi}, \\
\mathcal{F}_{\Delta}(\boldsymbol{r})=\mathcal{F}_{f}(\boldsymbol{r})+\mathcal{F}_{g}(\boldsymbol{r}), \\
\mathcal{F}_{f}(\boldsymbol{r})=\pi N_{0} \int \frac{d \theta}{2 \pi} T \sum_{\omega_{n}} \Delta^{*}(\boldsymbol{r}, \theta) f\left(\boldsymbol{r}, \theta, i \omega_{n}\right), \\
\mathcal{F}_{g}(\boldsymbol{r})=4 \pi N_{0} \int \frac{d \theta}{2 \pi} T \sum_{\omega_{n}>0}^{\omega_{c}} \int_{\omega_{n}}^{\omega_{c_{2}}} d \omega \\
\times \operatorname{Re}\{g(\boldsymbol{r}, \theta, i \omega)-1\},
\end{gathered}
$$

where $\mathcal{F}_{\Delta}(\boldsymbol{r})$ is the condensation energy density of the electron system and $\mathcal{F}_{H}(\boldsymbol{r})$ is the energy density of the magnetic field. We introduce an additional energy cutoff $\omega_{c_{2}}$ to evaluate the integration in Eq. (24). In this paper, we set $\omega_{c_{2}}=400 \Delta_{0}$ so that $\int d \boldsymbol{r} \mathcal{F}(\boldsymbol{r})$ reaches a converged value. The free-energy densities are normalized to $\mathcal{F}_{0}=N_{0}\left|\Delta_{0}\right|^{2} / 2$ which is the condensation energy density in a homogeneous $s$-wave superconductor. The 

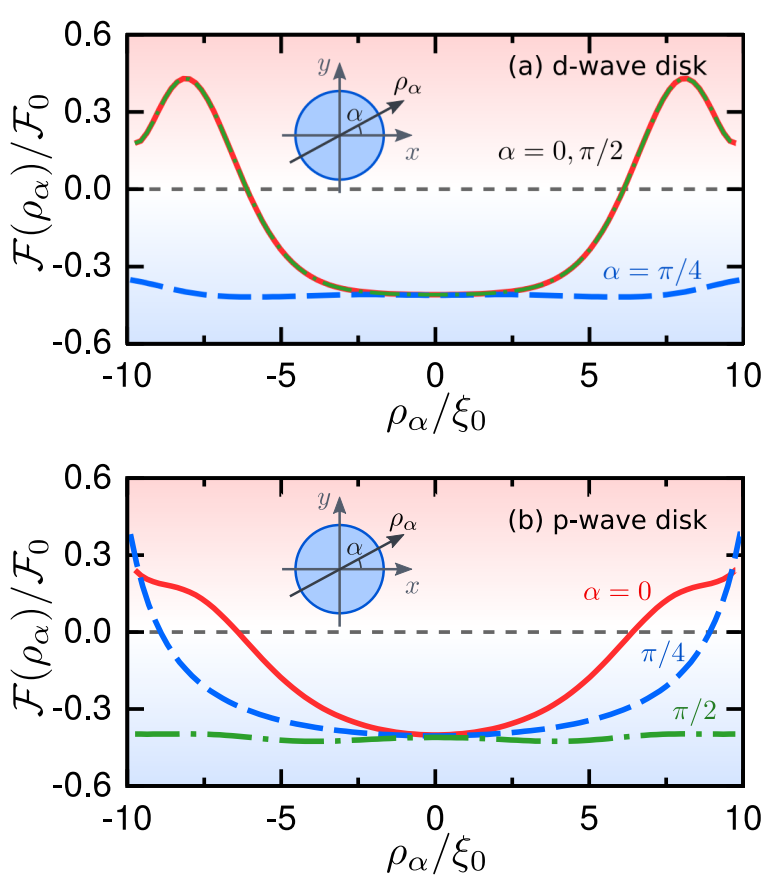

FIG. 8. (Color online) Free-energy densities on a trajectory $\rho_{\alpha}$ of a $d$-wave disk (a) and a $p$-wave disk (b), where $\mathcal{F}(\boldsymbol{r})$ is normalized to $\mathcal{F}_{0}=N_{0} \Delta_{0}^{2} / 2$. The angle $\alpha$ is measured from the $x$ axis as shown in schematics in the figures. The temperature is set to $T=0.1 T_{c}$ so that the superconducting disks show the paramagnetic response. The second energy cutoff is set to $\omega_{c_{2}}=400 \Delta_{0}$. The other parameters are fixed as $R=10 \xi_{0}, \lambda_{L}=5 \xi_{0}, \omega_{c}=10 \Delta_{0}$, and $H^{\mathrm{ext}}=0.01 H_{c_{1}}$.

temperature is set to be sufficiently low at $T=0.1 T_{c}$ so that the superconductor is in the paramagnetic phase.

The calculated results for the free-energy density for a $d$ wave and those for a $p$-wave disk are shown in Figs. 8(a) and $8(\mathrm{~b})$, respectively. The free-energy density is calculated along a trajectory $\rho_{\alpha}$ oriented at an angle $\alpha$ which is measured from the $x$ axis as shown in Fig. 8. In a $d$-wave disk, the results in Fig. 8(a) show that $\mathcal{F}(\boldsymbol{r})$ is negative around the disk center. However, it becomes positive near the surfaces at $\alpha=0$. On the other hand, $\mathcal{F}(\boldsymbol{r})$ at $\alpha=\pi / 4$ is almost flat and is always negative along the trajectory because odd-frequency pairs are absent in this direction. The results for $\alpha=\pi / 2$ are identical to those for $\alpha=0$ due to the fourfold symmetry. The free-energy density varies gradually from the line with $\alpha=0$ to that with $\alpha=\pi / 4$ as $\alpha$ increases from $\alpha=0$. The free energy of the whole disk $\int d \boldsymbol{r} \mathcal{F}(\boldsymbol{r})$ can be negative even though the disk is in the paramagnetic phase. In a $p$-wave disk, the increase of $\mathcal{F}(\boldsymbol{r})$ occurs only on two surfaces due to its $p$-wave symmetry. Therefore, we can conclude that both $d$ and $p$-wave superconducting states are more stable than the normal state even in the paramagnetic phase.

To analyze the details of the free-energy density further, we decompose the free-energy density at $\alpha=0$ into $\mathcal{F}_{H}$ and $\mathcal{F}_{\Delta}$ as shown in Fig. 9(a). When a superconductor shows a perfect Meissner effect, the energy of the magnetic field becomes $\mathcal{F}_{H}=\left(H^{\mathrm{ext}}\right)^{2} / 8 \pi$. In a small superconductor (i.e., $R \sim \lambda_{L}$ ), an external magnetic field penetrates into the whole disk. This suppresses $\mathcal{F}_{H}$ from $\left(H^{\text {ext }}\right)^{2} / 8 \pi$ at the center of the disk. Near the surface, on the other hand, paramagnetic odd-frequency
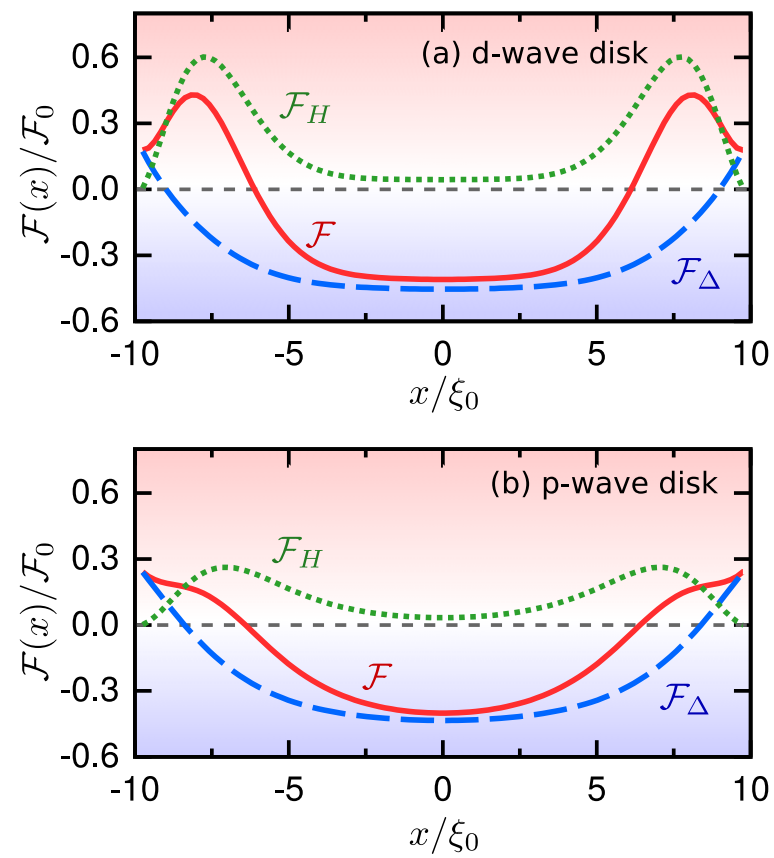

FIG. 9. (Color online) Condensation energy density and energy density of a magnetic field for $\alpha=0$. The results for a $d$-wave disk and those for a $p$-wave disk are plotted in (a) and (b), respectively. $\mathcal{F}, \mathcal{F}_{\Delta}$, and $\mathcal{F}_{H}$ are indicated by solid lines, broken lines, and dotted lines, respectively. All of the energy densities are normalized to $\mathcal{F}_{0}=$ $N_{0} \Delta_{0}^{2} / 2$, which is the condensation energy density of a homogeneous $s$-wave superconductor. All the parameters are set to be the same as those of Fig. 8 .

Cooper pairs attract the magnetic field, which increases $\mathcal{F}_{H}$ locally. The appearance of odd-frequency pairs also increases $\mathcal{F}_{\Delta}$ as discussed in the Appendix. As a result, $\mathcal{F}(\boldsymbol{r})$ becomes positive at the disk surface in both Figs. 9(a) and 9(b). The condensation energy $\mathcal{F}_{\Delta}$ is negative at the disk center, while, near the surface, $\mathcal{F}_{\Delta}$ increases due to the suppression of the pair potential there in both Figs. 9(a) and 9(b). In the Appendix, we analytically calculate the Green functions and the free-energy density near the surface of a semi-infinite $p_{x}$-wave superconductor. The result in Eq. (A21) indicates that the free-energy density is positive due to the appearance of odd-frequency Cooper pairs. The numerical results in Figs. 9(a) and 9(b) show that the free energy of the whole disk $\int d \boldsymbol{r} \mathcal{F}(\boldsymbol{r})$ remains negative because odd-frequency pairs are confined only near the surface. Therefore the paramagnetic superconducting state on $d$ - and $p$-wave disks is more stable than the normal state.

Next, we study the energetic properties of odd-frequency pairs. In our simulation, it is possible to obtain two superconducting states: a superconducting state in the absence of a magnetic field $H^{\text {ext }}=0$ and one in the presence of a magnetic field $H^{\text {ext }} \neq 0$. At $H^{\text {ext }}=0$, there is no electric current anywhere. Superconducting states at $H^{\text {ext }} \neq 0$, on the other hand, carry electric currents as shown in Figs. 5(a)-5(d). Here we compare the condensation energies of two such different superconducting states as shown in Fig. 10, where we plot $\mathcal{F}_{\Delta}$ on a $p$-wave disk for $\alpha=0$. The solid line and the broken line indicate results for $H^{\text {ext }} \neq 0$ and those 


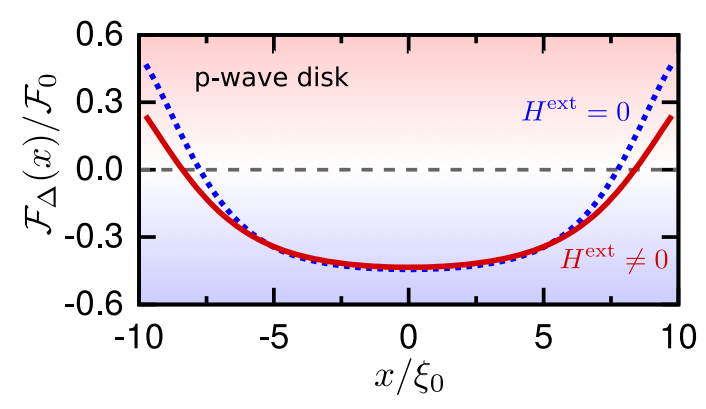

FIG. 10. (Color online) Condensation energy density $\mathcal{F}_{\Delta}$ obtained with an external field (solid line) and without an external field (dotted line). The calculations are carried out for a $p$-wave superconducting disk. All the parameters are set to be the same as those of Fig. 8.

for $H^{\text {ext }}=0$, respectively. As shown in Fig. $10, \mathcal{F}_{\Delta}$ near surfaces for $H^{\text {ext }} \neq 0$ is lower than that for $H^{\text {ext }}=0$. The odd-frequency pairing state in the presence of electric currents is more stable than that in the absence of electric currents. This energetic property explains the paramagnetic property of odd-frequency Cooper pairs. The argument above is valid also for a $d$-wave disk. In the Appendix, we present the analytical expression for the difference between the free energy at $H^{\text {ext }}=0$ and that at $H^{\text {ext }} \neq 0$. The results show that a magnetic field decreases the free energy at low temperature because odd-frequency Cooper pairs have the paramagnetic property.

\section{CONCLUSION}

We have theoretically studied the effects of surface roughness on the anomalous paramagnetic response of small unconventional superconducting disks by using the quasiclassical Green function method. We conclude that the paramagnetic property of $p$-wave superconductors is robust under surface roughness because $p$-wave superconductors host $s$-wave oddfrequency Cooper pairs at their surface. On the other hand, the paramagnetic property in $d$-wave superconductors is fragile in the presence of surface roughness. In this case, the oddfrequency pairs at the surface have $p$-wave orbital symmetry. We have also confirmed that the paramagnetic superconducting phase is more stable than the normal state by calculating the free energy.

\section{ACKNOWLEDGMENTS}

We are grateful to Y. Tanaka, S. Higashitani, Y. Nagato, N. Miyawaki, and S. Ikegaya for helpful discussion. This work was partially supported by "Topological Quantum Phenomena" (Grant No. 22103002) Grant-in Aid for Scientific Research on Innovative Areas, KAKENHI (Grant No. 26287069) from the Ministry of Education, Culture, Sports, Science and Technology (MEXT) of Japan, and by the Ministry of Education and Science of the Russian Federation (Grant No. 14Y.26.31.0007). S.-I.S. is supported in part by Grant-in-Aid for JSPS Fellows (Grant No. 15J00797) by Japan Society for the Promotion of Science (JSPS).

\section{APPENDIX: ANALYSIS IN A SEMI-INFINITE $p_{x}$-WAVE SUPERCONDUCTOR}

In a semi-infinite superconductor in two dimensions, it is possible to obtain an analytical expression for the Green functions in the clean limit. The evaluation of the electric current and free energy by using analytical expressions is helpful in understanding the numerical results in the text.

We assume that the superconductor occupies $x \geqslant 0$ and is uniform in the $y$ direction. A magnetic field is applied in the $z$ direction and its vector potential is given by $\boldsymbol{A}=A(x) \hat{\boldsymbol{y}}$. The Eilenberger equation in $2 \times 2 \mathrm{Nambu}$ space reads

$$
\begin{gathered}
i v_{F} \boldsymbol{k} \cdot \nabla \hat{g}+[\hat{H}, \hat{g}]=0, \\
\hat{g}\left(x, \boldsymbol{k}, i \omega_{n}\right)=\left[\begin{array}{cc}
g & f \\
s_{p} f & -g
\end{array}\right]_{\left(x, \boldsymbol{k}, i \omega_{n}\right)}, \\
\hat{H}=\left[\begin{array}{cc}
i \omega_{n}+e v_{F} \boldsymbol{k} \cdot \boldsymbol{A} & i \Delta(x, \boldsymbol{k}) \\
i s_{p} \underset{\sim}{\Delta}(x, \boldsymbol{k}) & -i \omega_{n}-e v_{F} \boldsymbol{k} \cdot \boldsymbol{A}
\end{array}\right], \\
s_{p}=\left\{\begin{array}{cc}
1, & \text { even parity, } \\
-1, & \text { odd parity, }
\end{array}\right.
\end{gathered}
$$

where the factor $s_{p}$ depends on the parity of the order parameter and the Green functions satisfy $g^{2}+s_{p} f f=1$. The Green functions can be expanded with respect to the vector potential as

$$
\begin{aligned}
g= & g^{(0)}+\left(-i e v_{F} \boldsymbol{k} \cdot \boldsymbol{A}\right) \partial_{\omega_{n}} g^{(0)} \\
& +\frac{1}{2}\left(-i e v_{F} \boldsymbol{k} \cdot \boldsymbol{A}\right)^{2} \partial_{\omega_{n}}^{2} g^{(0)}+\cdots, \\
f= & f^{(0)}+\left(-i e v_{F} \boldsymbol{k} \cdot \boldsymbol{A}\right) \partial_{\omega_{n}} f^{(0)} \\
& +\frac{1}{2}\left(-i e v_{F} \boldsymbol{k} \cdot \boldsymbol{A}\right)^{2} \partial_{\omega_{n}}^{2} f^{(0)}+\cdots,
\end{aligned}
$$

because a vector potential shifts the Matsubara frequency; here $g^{(0)}$ and $f^{(0)}$ are the Green functions in the absence of a vector potential. In what follows, we omit " (0)" from the Green function for simplicity. We note in Eq. (A6) that the parity and frequency symmetry of the second term on the right-hand side are opposite to those of the first term because $\boldsymbol{k}$ is an odd-parity function and $\partial_{\omega_{n}} f$ changes the frequency symmetry [13]. The imaginary part of an anomalous Green function represents a pairing correlation deformed by a vector potential.

In the case of a $p_{x}$-wave superconductor, it is possible to obtain a reasonable solution of the Eilenberger equation at $\boldsymbol{A}=\mathbf{0}$. When we assume the spatial dependence of the pair potential as

$$
\Delta(x, \theta)=\Delta(\theta) \tanh (x / \xi),
$$

with $\xi=v_{F} / \Delta_{0}$, the Green functions are represented by [27]

$$
\begin{gathered}
g\left(x, \theta, i \omega_{n}\right)=\frac{\omega_{n}}{\Omega}+\frac{\Delta^{2}(\theta)}{2 \omega_{n} \Omega} \cosh ^{-2}\left(\frac{x}{\xi}\right), \\
f_{P}\left(x, \theta, i \omega_{n}\right)=\frac{\Delta(\theta)}{\Omega} \tanh \left(\frac{x}{\xi}\right),
\end{gathered}
$$




$$
f_{I}\left(x, \theta, i \omega_{n}\right)=-\frac{\Delta^{2}(\theta)}{2 \omega_{n} \Omega} \cosh ^{-2}\left(\frac{x}{\xi}\right),
$$

where $\Omega=\left[\omega_{n}^{2}+\Delta^{2}(\theta)\right]^{1 / 2}$ and $\Delta(\theta)=\Delta_{0} \cos (\theta)$. The Green function $f_{P}$ represents the principal pairing correlation in the bulk state, whereas $f_{I}$ represents the pairing correlation induced by a surface at $x=0$. They are calculated from the anomalous Green function as

$$
\begin{gathered}
f_{P}\left(x, \theta, i \omega_{n}\right)=\left.\frac{1}{2}\left(f+s_{p} \underset{\sim}{f}\right)\right|_{\left(x, \theta, i \omega_{n}\right)}, \\
f_{I}\left(x, \theta, i \omega_{n}\right)=\left.\frac{1}{2}\left(f-s_{p} \underset{\sim}{f}\right)\right|_{\left(x, \theta, i \omega_{n}\right)} .
\end{gathered}
$$

Deep inside the superconductor (i.e., $x \gg \xi$ ), we obtain $f_{P}=$ $f$ and $f_{I}=0$.

\section{Current density}

From the expression for the electric current in Eq. (12), we define a linear response function $\mathcal{R}_{\mu, \nu}$ by

$$
\begin{gathered}
j_{\mu}(\boldsymbol{r})=-\frac{e^{2}}{m} \mathcal{R}_{\mu, \nu} A_{\nu}, \\
\frac{\mathcal{R}_{\mu, v}}{n_{e}}=4 \pi T \sum_{\omega_{n}} \int \frac{d \theta}{2 \pi} k_{\mu} k_{\nu} \partial_{\omega_{n}} g\left(\boldsymbol{r}, \theta, i \omega_{n}\right)
\end{gathered}
$$

with $\boldsymbol{k}=(\cos \theta, \sin \theta)$ and $n_{e}=v_{F}^{2} N_{0} m$ the electron density in two dimensions. The diagonal elements of the response function $\mathcal{R}_{\mu, \mu}$ correspond to the so-called pair density. In the present situation, by substituting Eq. (A8) into Eq. (A14), we obtain

$$
\begin{gathered}
\frac{\mathcal{R}_{y, y}}{n_{e}}=1-\kappa_{1} \frac{\Delta_{0}}{\omega_{0}} \cosh ^{-2}\left(\frac{x}{\xi}\right), \\
\kappa_{1}=\int \frac{d \theta}{2 \pi} \sin ^{2}(\theta)|\cos (\theta)|=\frac{2}{3 \pi},
\end{gathered}
$$

where $\omega_{0}=\pi T$ is the low-energy cutoff in the Matubara summation. Using the normalization condition, the integrand in Eq. (A14) can be represented in an alternative way,

$$
\partial_{\omega_{n}} g=\left[-f_{P} \partial_{\omega_{n}} f_{P}+f_{I} \partial_{\omega_{n}} f_{I}\right] / g
$$

It is possible to confirm that $-f_{P} \partial_{\omega_{n}} f_{P} / g$ corresponds to the first term in Eq. (A15), whereas $f_{I} \partial_{\omega_{n}} f_{I} / g$ contributes to the second term. In this way, we can confirm that induced oddfrequency Cooper pairs indicate a paramagnetic response to an external magnetic field. Equation (A15) suggests that the paramagnetic response is stronger at a lower temperature.

\section{Free-energy density}

Substituting Eqs. (A8)-(A10) into Eqs. (22)-(24), we find the free-energy density at $\boldsymbol{A}=\mathbf{0}$,

$$
\begin{gathered}
\mathcal{F}_{f}=N_{0} \kappa_{2} \Delta_{0}^{2} \ln \left(\frac{2 \omega_{c}}{\Delta_{0}}\right) \tanh ^{2}\left(\frac{x}{\xi}\right), \\
\mathcal{F}_{g}=-N_{0} \kappa_{2} \Delta_{0}^{2} \ln \left(\frac{2 \omega_{c}}{\Delta_{0}}\right) \tanh ^{2}\left(\frac{x}{\xi}\right) \\
+N_{0} \Delta_{0}^{2} \kappa_{2}\left[\cosh ^{-2}\left(\frac{x}{\xi}\right)-\frac{1}{2}\right], \\
\kappa_{2}=\int \frac{d \theta}{2 \pi} \cos ^{2}(\theta)=\frac{1}{2} .
\end{gathered}
$$

As a result, we obtain

$$
\mathcal{F}_{\Delta}=N_{0} \Delta_{0}^{2} \kappa_{2}\left[\cosh ^{-2}\left(\frac{x}{\xi}\right)-\frac{1}{2}\right] .
$$

The free-energy density becomes positive at $x=0$ due to the appearance of odd-frequency pairs.

The contribution of a magnetic field to the free energy can be evaluated by applying the expansion in Eqs. (A5) and (A6) to Eqs. (A8)-(A10). Within a second-order expansion, we find that both the pair potential obtained by Eq. (9) and $\mathcal{F}_{f}$ remain unchanged. The second-order correction to $\mathcal{F}_{g}$ is given by

$$
\mathcal{F}_{g}^{(2)}=\frac{1}{2} N_{0}\left[\operatorname{ev}_{F} A(x)\right]^{2}\left[1-\kappa_{1} \frac{\Delta_{0}}{\omega_{0}} \cosh ^{-2}\left(\frac{x}{\xi}\right)\right] .
$$

The results indicate that $\mathcal{F}_{g}^{(2)}$ can be locally negative (paramagnetic) at low enough temperature. This explains the decrease of the free energy in a magnetic field shown in Fig. 10. We also obtain the electric current in Eqs. (A13) and (A15) from $j_{y}(x)=-\partial \mathcal{F} / \partial A_{y}$.
[1] See, for example, M. Tinkham, Introduction to Superconductivity, 2nd ed. (McGraw-Hill, New York, 1996).

[2] W. Braunisch, N. Knauf, V. Kataev, S. Neuhausen, A. Grutz, A. Kock, B. Roden, D. Khomskii, and D. Wohlleben, Phys. Rev. Lett. 68, 1908 (1992).

[3] B. Schliepe, M. Stindtmann, I. Nikolic, and K. Baberschke, Phys. Rev. B 47, 8331 (1993).

[4] D. J. Thompson, M. S. M. Minhaj, L. E. Wenger, and J. T. Chen, Phys. Rev. Lett. 75, 529 (1995).

[5] A. K. Geim, S. V. Dubonos, J. G. S. Lok, M. Henini, and J. C. Maan, Nature (London) 396, 144 (1998).

[6] P. Visani, A. C. Mota, and A. Pollini, Phys. Rev. Lett. 65, 1514 (1990).
[7] A. C. Mota, P. Visani, A. Pollini, and K. Aupke, Physica B 197, 95 (1994).

[8] Y. Asano, A. A. Golubov, Ya. V. Fominov, and Y. Tanaka, Phys. Rev. Lett. 107, 087001 (2011).

[9] S. Mironov, A. Melnikov, and A. Buzdin, Phys. Rev. Lett. 109, 237002 (2012).

[10] T. Yokoyama, Y. Tanaka, and N. Nagaosa, Phys. Rev. Lett. 106, 246601 (2011).

[11] A. S. Melnikov, A. V. Samokhvalov, S. M. Kuznetsova, and A. I. Buzdin, Phys. Rev. Lett. 109, 237006 (2012).

[12] Y. Asano, M. Ozaki, T. Habe, A. A. Golubov, and Y. Tanaka, Phys. Rev. B 86, 024510 (2012). 
[13] Y. Asano, Y. V. Fominov, and Y. Tanaka, Phys. Rev. B 90, 094512 (2014).

[14] Y. Tanaka and A. A. Golubov, Phys. Rev. Lett. 98, 037003 (2007).

[15] Y. Tanaka, Y. Tanuma, and A. A. Golubov, Phys. Rev. B 76, 054522 (2007).

[16] V. L. Berezinskii, Pis'ma Zh. Eksp. Teor. Fiz. 20, 628 (1974) [JETP Lett. 20, 287 (1974)].

[17] F. S. Bergeret, A. F. Volkov, and K. B. Efetov, Phys. Rev. Lett. 86, 4096 (2001); Rev. Mod. Phys. 77, 1321 (2005).

[18] S.-I. Suzuki and Y. Asano, Phys. Rev. B 89, 184508 (2014).

[19] H. Walter, W. Prusseit, R. Semerad, H. Kinder, W. Assmann, H. Huber, H. Burkhardt, D. Rainer, and J. A. Sauls, Phys. Rev. Lett. 80, 3598 (1998).

[20] A. Poenicke, Yu. S. Barash, C. Bruder, and V. Istyukov, Phys. Rev. B 59, 7102 (1999).

[21] A. Zare, A. Markowsky, T. Dahm, and N. Schopohl, Phys. Rev. B 78, 104524 (2008).

[22] Yu. S. Barash, M. S. Kalenkov, and J. Kurkijarvi, Phys. Rev. B 62, 6665 (2000).

[23] Y. Tanaka, Y. Tanuma, and S. Kashiwaya, Phys. Rev. B 64, 054510 (2001).

[24] A. M. Black-Schaffer, D. S. Golubev, T. Bauch, F. Lombardi, and M. Fogelström, Phys. Rev. Lett. 110, 197001 (2013).

[25] G. Eilenberger, Z. Phys. 214, 195 (1968).

[26] N. Schopohl and K. Maki, Phys. Rev. B 52, 490 (1995).

[27] N. Schopohl, arXiv:cond-mat/9804064.

[28] M. Eschrig, Phys. Rev. B 80, 134511 (2009).

[29] Y. Nagai, K. Tanaka, and N. Hayashi, Phys. Rev. B 86, 094526 (2012).
[30] M. Ichioka, N. Hayashi, N. Enomoto, and K. Machida, Phys. Rev. B 53, 15316 (1996). The authors considered a $d$-wave superconductor with a vortex without any surface. When a vortex with a nonzero winding number exists, the pair potential must be zero at the vortex core to remove a singularity from the pair potential. Therefore the amplitude of the pair potential around the vortex core is basically isotropic in real space. In our simulation, on the other hand, the formation of Andreev bound states at the surface suppressed the pair potential there. The pair potential is suppressed for different reasons in the two cases.

[31] The sign change in the pair potential in the normal reflection process by a surface plays an essential role in forming surface Andreev bound states. Let us assume that $k_{x}\left(k_{y}\right)$ is the wave number in the direction perpendicular (parallel) to the surface. The pair potential on the way to the surface (on the way back from the surface) is $\Delta\left(k_{x}, k_{y}\right)\left[\Delta\left(-k_{x}, k_{y}\right)\right]$ because the wave number in the direction perpendicular to the surface changes its sign in normal reflection. The Andreev bound state appears when $\Delta\left(k_{x}, k_{y}\right) \Delta\left(-k_{x}, k_{y}\right)<0$ is satisfied, as shown by Y. Asano, Y. Tanaka, and S. Kashiwaya, Phys. Rev. B 69, 134501 (2004). In a $d_{x y}$-wave superconductor with a pair potential $\Delta k_{x} k_{y}$, the Andreev bound states appear in four surfaces in the $x$ and $y$ directions (nodal directions). In a $p_{x}$-wave superconductor with $\Delta k_{x}$, two surfaces in the $x$ direction (antinodal directions) host the Andreev bound states.

[32] Y. Asano and Y. Tanaka, Phys. Rev. B 65, 064522 (2002).

[33] S. Ikegaya, Y. Asano, and Y. Tanaka, Phys. Rev. B 91, 174511 (2015).

[34] G. Eilenberger, Z. Phys. 190, 142 (1966). 